\title{
Photoinduced Charge-Transfer Reactions of Tricyanoethlene with Aromatic Hydrocarbon Electron Donors. I. Emission Studies of Naphthalene-Tricyanoethylene Exciplex
}

\author{
J. Dresner, K. Kwapisz, I. Deperasińska, J. Prochorow \\ Institute of Physics, Polish Academy of Sciences \\ al. Lotników 32/46, 02-668 Warsaw, Poland \\ P. Bruni, A. Giardina and G. Tosi \\ Dipartimento di Scienze dei Materiali e della Terra \\ Universita degli Studi di Ancona \\ via Brecce Bianche, 60131 Ancona, Italy
}

(Received March 11, 2002; in final form June 29, 2002)

\begin{abstract}
The fluorescence quenching of a series of aromatic hydrocarbon electron donors by an electron acceptor tricyanoethylene has been observed. The quenching mechanism is consistent with the charge-transfer (or electron-transfer) mechanism, as indicated by the appearance of a new, broad, and structureless exciplex emission band. Detailed, steady-state, and time-resolved emission studies of the kinetics and thermodynamics of exciplex formation and relaxation have been performed for naphthalene-tricyanoethylene system. It was found that the kinetics of monomer-exciplex equilibrium for this system, in a non-polar solvent ( $n$-hexane), can be described in terms of a simple two-state photokinetic scheme. Within such a scheme, under conditions of the present experiment, napthalene-tricyanoethylene system approaches the limits under which exciplex formation is very effective - it becomes exclusively diffusion-controlled and at the same time a competitive process of thermal dissociation of exciplex ceases to operate. This leads to a very rarely observed reversal of physical meaning of the decay parameters which are describing the rise and the decay of exciplex fluorescence.
\end{abstract}

PACS numbers: 82.30.Fi, 82.60.Hz, 32.50.-j 


\section{Introduction}

The importance of the process of photoinduced transfer of charge (or electron) as an initial step in quenching of fluorescence of aromatic hydrocarbons by various organic electron acceptors in liquid solutions has been recognized at the beginning of sixties of last century $[1,2]$ and pretty soon the photokinetic scheme of the formation of excited-state complex — an exciplex has been proposed [3-5]. In such a diffusion-controlled (in solution) bimolecular quenching reaction between an electron donor (D) and electron acceptor (A):

$$
\mathrm{D}+h \nu_{\mathrm{D}} \rightarrow \mathrm{D}^{*}+\mathrm{A} \rightarrow\left(\mathrm{D}^{+} \mathrm{A}^{-}\right)^{*}
$$

an encounter of a primarily excited species (either donor $\mathrm{D}^{*}$ or acceptor $\mathrm{A}^{*}$ ) with its counterpart (of a given electron-donor-acceptor system under consideration) in the ground state (either A or D) leads to the transfer of charge (electron) between interacting molecules and to the creation of excited $\left(\mathrm{D}^{+} \mathrm{A}^{-}\right)^{*}$ charge transfer $(\mathrm{CT})$ state - an exciplex.

The detailed studies have since been made with the use of practically all experimental techniques of emission spectroscopy which were developed in the period of last forty years (for a review see [6-8]). In recent years the main effort has been concentrated on sophisticated time-resolved femtosecond emission studies of the dynamics of electron transfer processes and relaxation of excited CT state and on elaborate theoretical interpretations of such experiments [9-13]. The main object of these advanced studies was a ground-state-stable electron-donor-acceptor (EDA) complex formed between tetracyanoethlene (TCNE) and hexamethylbenzene (HMB). This is not surprising, as TCNE is one of the strongest electron acceptors and its molecular complexes with electron donors from the family of methyl-substituted derivatives of benzene (and with other aromatic hydrocarbons) were known from the very beginning of studies of EDA complexes [14] — known also, after the formulation by Mulliken of his resonance theory of CT state [15], as the charge-transfer, CT complexes. Hence the parameters of the ground-state equilibria:

$$
\mathrm{D}+\mathrm{A} \rightleftharpoons(\mathrm{DA})
$$

of CT complexes of TCNE, as well as their absorption spectra, connected with:

$$
(\mathrm{DA})+h \nu_{\mathrm{CT}} \rightarrow\left(\mathrm{D}^{+} \mathrm{A}^{-}\right)^{*}
$$

electronic transition, were studied in great details, catalogued (see for instance [16]) and often exploited in the discussions of various aspects of CT interactions.

Almost from the very beginning, the CT complexes of TCNE were known as non-fluorescent in liquid solutions, neither upon excitation within the characteristic absorption band (the charge-transfer, CT, band) of CT complex, nor upon excitation within the absorption bands of its molecular components (either D or A). And only recently a steady-state fluorescence spectrum of TCNE-HMB 
complex in liquid solution in the near-infrared has been reported [17]. On the other hand, their CT fluorescence is very efficient in rigid media [18-20] and can also be observed in a supersonic jet expansion [21]. It is rather well established now that the lack of CT fluorescence (fluorescence excited within the CT absorption band of the complex) of TCNE complexes is due to the efficient non-radiative processes connected with different relaxation pathways from excited Franck-Condon states of different orientational isomers to the equilibrated CT state [19, 22], while in polar solvents excitation of the complex results in efficient and very fast formation of separated (solvated) ion pairs: $(\mathrm{DA})+h \nu_{\mathrm{CT}} \rightarrow \mathrm{D}_{\mathrm{s}}^{+} \ldots \mathrm{A}_{\mathrm{s}}^{-}[23]$.

The efficiency of TCNE as a quencher of fluorescence of all aromatic electron donors seems to be very high, even when compared with other strong electron acceptors (e.g. 1,2,4,5-tetracyanobenzene, TCNB) and must be related to its high chemical reactivity as a strong electrophilic reagent and its early recognized versatile applications in cyanocarbon chemistry [24]. Contrary to the very rich database available for TCNE, almost nothing, or very little is known about CT complexes and exciplexes of other members of cyanoethylene family, i.e.: mono-substituted acrylonitrile, two dicyanoethylenes (i.e. maleonitrile and fumaronitrile), and tricyanoethylene. This is connected in part with the increasing order of the energy of the first electronic transition in cyanoethylenes family, from tetra- to mono-substituted cyanoethylenes (and thus reversed order of ability toward CT complex formation with a given electron donor), which makes difficult spectroscopic investigations (from near to far $\mathrm{UV}$ ) of the ground state $\mathrm{D}+\mathrm{A} \rightleftharpoons(\mathrm{DA})$ association equilibria. Another factor is connected with the fact that other electron acceptors of cyanoethylenes family are considerably less stable chemically (and photochemically) than TCNE. The formation of exciplexes, as verified by the appearance of exciplex fluorescence in various solvents, has been reported only for fumaronitrile (trans-1,2-dicyanoethylene) and a series of polycondensed aromatic hydrocarbons [25]. In the case of tricyanoethylene (TRCNE) the formation of its weak $\mathrm{CT}$ complexes with toluene and durene in $\mathrm{CH}_{2} \mathrm{Cl}_{2}$ solution has been mentioned on the occasion of its first synthesis [26].

In this paper we report first observations of fluorescence quenching reactions (1) of aromatic hydrocarbon donors by TRCNE in solution and the formation of relevant exciplexes. A detailed analysis of the results of steady-state and time-resolved emission studies were carried out for exciplex of naphthalene with TRCNE. This system, as formerly inferred from investigations of the ground-state association equilibria is capable to form a weak ground-state-stable CT complex [27]. Present studies of its excitation spectra of exciplex fluorescence (cf. Sect. 4.2) are unequivocally confirming this fact. Furthermore, investigations of the kinetics of its total (monomer and exciplex) emission, show that the formation and relaxation of naphthalene-TRCNE exciplex follows a simple two-state photokinetic scheme corresponding to diffusion-controlled bimolecular fluorescence quenching reaction (1). Within this photokinetic scheme, the kinetics of monomer-exciplex 
equilibrium of naphthalene-TRCNE system reaches the limit at which the physical meaning of the decay parameters of exciplex emission becomes reversed.

\section{Kinetic scheme for exciplex formation and decay}

An exciplex formation reaction and its decay can be described by the following, simplified photokinetic scheme [2]:

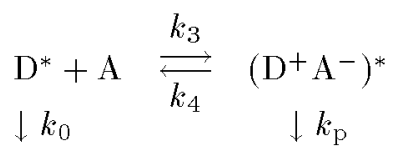

provided that its naturally stepwise character can be neglected (in the first approximation), or in other words, that the diffusion-controlled close encounter of $\mathrm{D}^{*}$ and $\mathrm{A}$ in a solution [cf. reaction scheme (1)], which results in the formation of not well-defined transient species [or encounter complex $\left(D^{*} A\right)$ ] has a probability $p$ toward formation of exciplex $\left(\mathrm{D}^{+} \mathrm{A}^{-}\right)^{*}$, and thus the rate constant (second order) of exciplex formation is $k_{3} \equiv p \cdot k_{\text {diff }}$.

The rates of other processes operating in photokinetic scheme (4) are following:

- $k_{0}=\left(k_{1}+k_{2}\right)$ is the sum of radiative $\left(k_{1}\right)$ and radiationless $\left(k_{2}\right)$ decay rate constants of primarily excited monomer (the donor),

- $k_{4}$ is the rate constant of thermal dissociation of exciplex that regenerates $\mathrm{D}^{*}$ and A species (known also as the "feedback dissociation" of exciplex),

$-k_{\mathrm{p}}=\left(k_{5}+k_{6}\right)$ is the sum of radiative $\left(k_{5}\right)$ and radiationless $\left(k_{6}\right)$ decay rate constants of exciplex.

Observations of the concentration and temperature behavior of the fluorescence intensities of both (and of the two only) emitting species involved in such a photokinetic scheme should eventually lead to determination of the rate constants of all process operating within the scheme. And this is an ultimate test of validity of any proposed photokinetic scheme in any particular case considered. The changes of the concentration of both excited species, $\left[D^{*}\right]$ and $\left[\left(D^{+} A^{-}\right)^{*}\right]$, which are coupled by the scheme (4), are given by the pair of coupled ordinary differential equations [28]:

$$
\begin{aligned}
& \mathrm{d}\left[\mathrm{D}^{*}\right] / \mathrm{d} t=I_{a}(t)+k_{4}\left[\left(\mathrm{D}^{+} \mathrm{A}^{-}\right)^{*}\right]-\left(k_{1}+k_{2}+k_{3}[\mathrm{~A}]\right)\left[\mathrm{D}^{*}\right], \\
& \mathrm{d}\left[\left(\mathrm{D}^{+} \mathrm{A}^{-}\right)^{*}\right] / \mathrm{d} t=k_{3}[\mathrm{~A}]\left[\mathrm{D}^{*}\right]-\left(k_{4}+k_{\mathrm{p}}\right)\left[\left(\mathrm{D}^{+} \mathrm{A}^{-}\right)^{*}\right] .
\end{aligned}
$$

Since, the primary excitation of $\mathrm{D}$ can be either time-independent, $I_{a}$, or time-dependent, $I_{a}(t)$, the photokinetic scheme can be investigated under both, the steady-state (photostationary) and time-resolved (transient) experimental conditions. 
In the practical analysis of experimental results it must be taken care of some additional effects not directly included into the photokinetic scheme (4), and by the same token not included into the population equations (5). As we mentioned above in Sec. 1, most of EDA systems (and particularly those with strong electron acceptors) are capable of the formation of ground-state-stable CT complexes and hence an excited CT state can also be created upon excitation within the CT absorption band [cf. reactions (2) and (3)]. If, in such a case, the absorption bands of monomer ( $h \nu_{\mathrm{D}}$ transition in donor molecule) and of CT complex ( $h \nu_{\mathrm{CT}}$ transition in CT complex) are overlapping, then the kinetic scheme (4) is of limited validity (or not applicable at all). Although, in nonpolar (or of medium polarity) liquid solutions, an equilibrated $\left(\mathrm{D}^{+} \mathrm{A}^{-}\right)^{*}$ state is independent of the way of its creation [29], its dynamics of formation via both routes [reaction schemes (1) and (3), respectively] is essentially different. It should also be taken care of static quenching of $\mathrm{D}^{*}$ fluorescence, which is connected with direct excitation of higher states of (DA) complex, i.e. with local excitation of D bound within the CT complex in its ground state.

\section{Experimental procedures}

Tricyanoethylene was synthesized (at Universita degli Studi di Ancona) following the five-step synthesis procedure described in [25]. After preliminary purification (by chromatography and recrystallization) it was stored at low temperature $\left(-30^{\circ} \mathrm{C}\right)$. Directly before the use TRCNE was sublimed in vacuo and its pale orange-yellow crystals were used for sample preparation (in $n$-hexane solution it was stable for several days, when kept in the dark). Electron donors were purified by distillation ( $p$-xylene), sublimation in vacuo (hexamethylbenzene) or by zone-refining (naphthalene). Solvents from Merck (Uvasol for spectroscopy) were used without further purification. All liquid samples were degassed by bubbling with $\mathrm{Ar}$ gas (for $\sim 30 \mathrm{~min}$ prior to the measurements).

For steady-state emission measurements the home-computerized commercial Perkin-Elmer 512 spectrofluorimeter was used.

For time-resolved and decays measurements, a subtractive double $0.25 \mathrm{~m}$ monochromator (CVI) was used. For excitation of fluorescence a double-jet dye laser (Coherent 702-CD) tunable in 285-310 nm (SHG of Rhodamine 6G dye), synchronously pumped by a mode-locked, cavity dumped Nd:YAG laser (Coherent Antares 76) was used. Fluorescence was detected with the use of a time-correlated single photon counting (TCSPC) equipped with XP 2020 Phillips photomultiplier with the effective temporal resolution of $c a .200$ ps. The decay parameters were deconvoluted from the decay curves with the aid of home-written software (least-square fitting) allowing up to the three-exponential analysis. 


\section{Experimental results: analysis and discussion}

\subsection{Steady-state fluorescence and fluorescence excitation spectra of TRCNE-aromatic hydrocarbon systems}

Absorption of TRCNE in $n$-hexane solution starts in near UV range and, as illustrated in Fig. 1, its first absorption band, with a maximum located at $\sim 240 \mathrm{~nm}$, is practically lacking any vibrational structure (it has a shoulder at $255 \mathrm{~nm}$ ). As seen in Fig. 1, an optical excitation within this absorption band leads to the observation of fluorescence of TRCNE. A very weak and wide fluorescence band of TRCNE is also lacking any vibrational features and has a maximum at ca. $285 \pm 3 \mathrm{~nm}$.

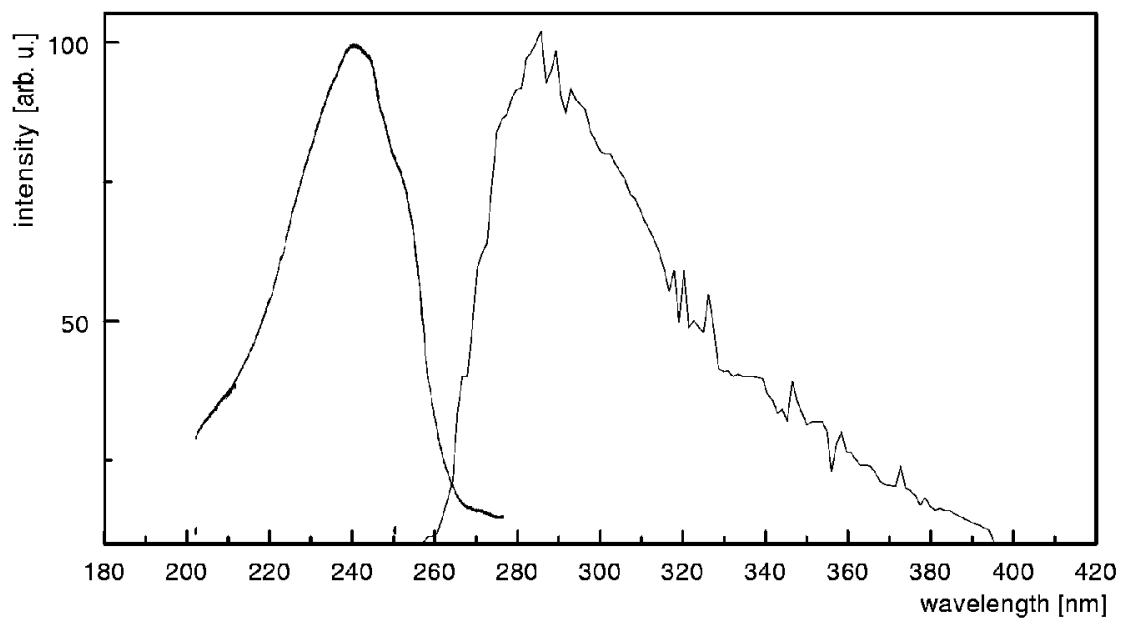

Fig. 1. Absorption and fluorescence spectra of TRCNE in $n$-hexane solution at room temperature.

Figures 2-4 are illustrating the basic experimental fact of present studies it is found that in liquid solution of $n$-hexane, fluorescence of three aromatic hydrocarbon donors, i.e. of $p$-xylene, naphthalene, and hexamethylbenzen (excited in their respective molecular absorption bands) is being quenched upon an addition of TRCNE to their solutions. Furthermore it is clearly seen that the addition of TRCNE always results in the appearance of a new, broad, and structureless emission band located on the low-energy side of aromatic hydrocarbon fluorescence band. In a manner typical of bimolecular fluorescence quenching reaction (with an exciplex formation), the intensity of this new fluorescence band (although very low) is increasing with increasing concentration of quencher (TRCNE) at the expense of intensity of quenched fluorescence of aromatic hydrocarbon.

A spectral position of this new fluorescence band (in $n$-hexane solution) is: $\sim 500, \sim 510$, and $\sim 590 \mathrm{~nm}$ in the case of $p$-xylene, naphthalene, and hexamethylbenzen, respectively, and it correlates with the ionization potential of the aromatic 


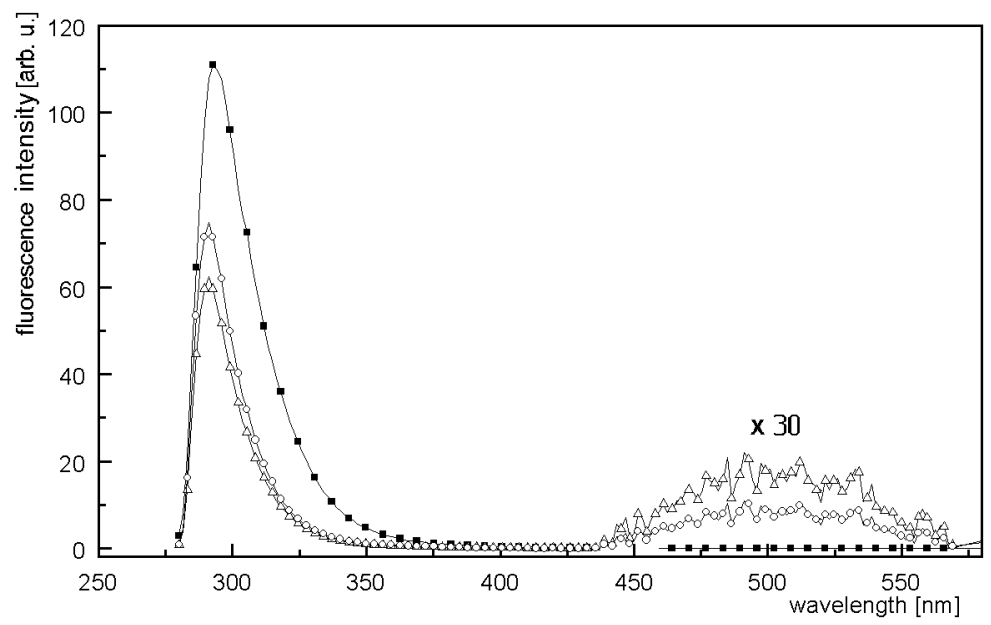

Fig. 2. Concentration dependence of the fluorescence spectrum of $p$-xylene - TRCNE in $n$-hexane solution at room temperature. Concentration of $p$-xylene is constant $3.2 \times 10^{-1} \mathrm{M}$. Concentration of TRCNE is: 0 (TRCNE solution only, full squares), $3 \times 10^{-4} \mathrm{M}$ (circles) and $6 \times 10^{-4} \mathrm{M}$ (triangles). Fluorescence was excited within the $p$-xylene absorption band at $280 \mathrm{~nm}$.

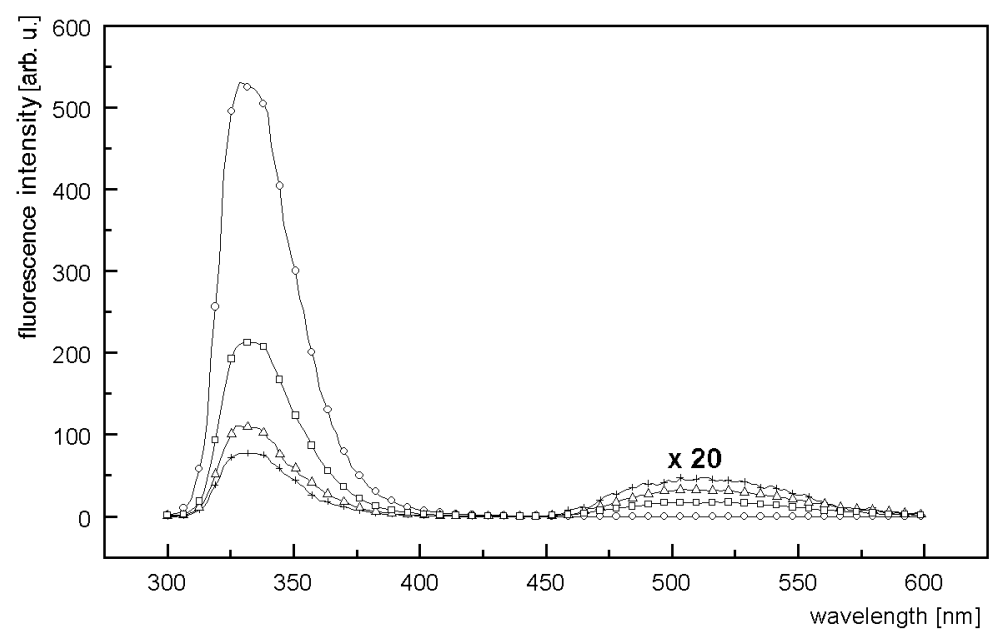

Fig. 3. Concentration dependence of the fluorescence spectrum of naphthalene-TRCNE in $n$-hexane solution at room temperature. Concentration of naphthalene is constant $4 \times 10^{-3} \mathrm{M}$. Concentration of TRCNE is: 0 (TRCNE solution only, circles), $2 \times 10^{-4} \mathrm{M}$ (squares), and $6 \times 10^{-4} \mathrm{M}$ (triangles) and $8 \times 10^{-4} \mathrm{M}$ (crosses). Fluorescence was excited within the naphthalene absorption band at $298 \mathrm{~nm}$.

hydrocarbon donor (which decreases from $8.4,8.14$, and $7.85 \mathrm{eV}$ for $p$-xylene, naphthalene, and hexamethylbenzen, respectively). Such a correlation serves as a first 


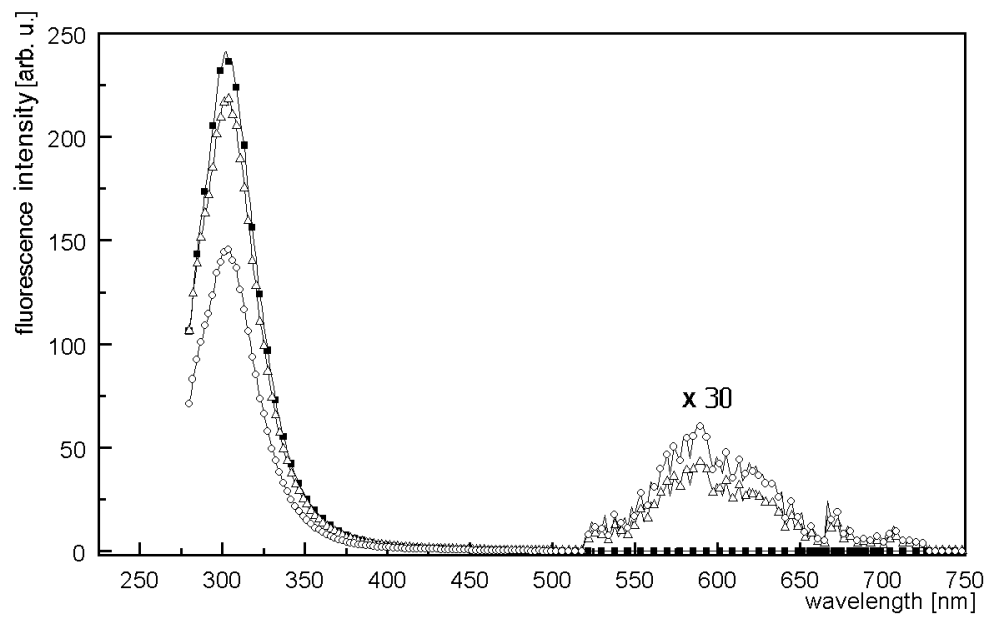

Fig. 4. Concentration dependence of the fluorescence spectrum of hexamethylbenzen-TRCNE in $n$-hexane solution at room temperature. Concentration of hexamethylbenzene is constant $-4 \times 10^{-3} \mathrm{M}$. Concentration of TRCNE is: 0 (TRCNE solution only, full squares), $6 \times 10^{-4} \mathrm{M}$ (triangles), and $8 \times 10^{-4} \mathrm{M}$ (circles). Fluorescence was excited within the hexamethylbenzene absorption band at $275 \mathrm{~nm}$.

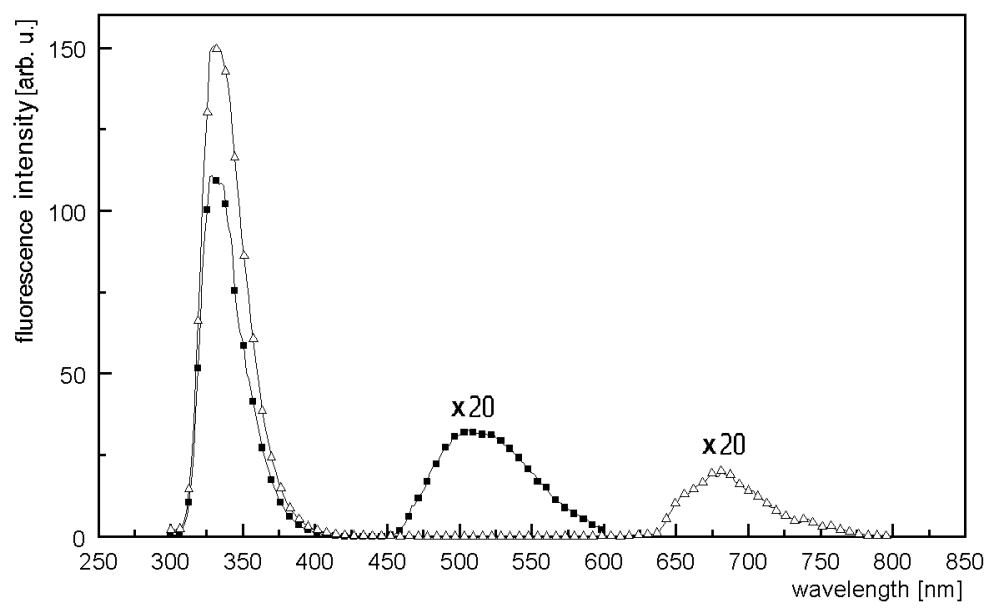

Fig. 5. Fluorescence spectra of naphthalene-TRCNE in different solvents: $n$-hexane (full squares) and dichloroethane (triangles). Fluorescence was excited within the naphthalene absorption band at $298 \mathrm{~nm}$.

and firm indication of the charge-transfer character of excited state which is initial for the new emission band observed in solutions of a series of electron donors with a given electron acceptor (TRCNE in this case). This fluorescence band shows also a remarkable sensitivity to the solvent polarity. As illustrated in Fig. 5, for 
TRCNE-napthalene system, upon going from non-polar solvent ( $n$-hexane, with dielectric constant $\varepsilon=1.89)$ to polar solvent (1,2-dichloroetane, with $\varepsilon=9.6$ ), a huge shift of this band: from $510 \mathrm{~nm}$ in $n$-hexane to $\sim 680 \mathrm{~nm}$ in dichloroethane (with no change in the position of the monomer, donor fluorescence band in both solvents) is observed. And this once again reveals strongly polar character of excited state of emitting species.

All listed-above observations lead to the conclusion that the new emission band which is observed in these systems must be attributed to the fluorescence of $\left(\mathrm{D}^{+} \mathrm{A}^{-}\right)^{*}$ exciplex, formed between electron acceptor (TRCNE) and excited aromatic hydrocarbon donor, according to diffusion- controlled reaction scheme (1).

An additional important piece of information comes from investigations of fluorescence excitation spectra of exciplex fluorescence. This is demonstrated in Fig. 6, which illustrates exciplex fluorescence excitation spectrum of naphthalene-TRCNE system (monitored at $510 \mathrm{~nm}$, i.e. at the maximum of exciplex fluorescence band, cf. Fig. 3). It is clearly seen that in this excitation spectrum, besides an expected absorption band of napthalene (donor), another absorption band shows up in the long-wavelength part of excitation spectrum. This very weak and very

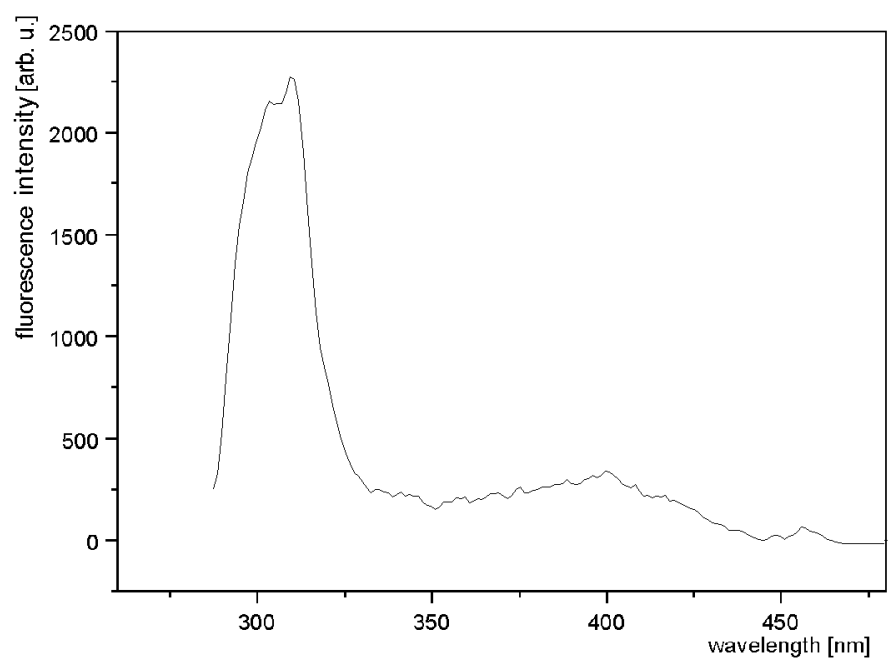

Fig. 6. Fluorescence excitation spectrum of naphthalene-TRCNE in $n$-hexane solution at room temperature, monitored at maximum of exciplex fluorescence band $(510 \mathrm{~nm})$.

broad absorption band, located at ca. $400 \pm 10 \mathrm{~nm}$, which gives rise to the observation of exciplex-like fluorescence of naphthalene-TRCNE electron-donor-acceptor system, is not connected with the fluorescence quenching mechanism described within the framework of reaction scheme (1). There is no doubt that this is an inherent CT absorption band of CT complex of naphthalene-TRCNE formed and stable in the ground state and described in terms of ground-state equilibrium (2). 
Direct excitation in this absorption band gives rise to CT fluorescence from the excited $\left(\mathrm{D}^{+} \mathrm{A}^{-}\right)^{*} \mathrm{CT}$ state. We find once again that in this case, as for many other EDA systems, the excited $\left(\mathrm{D}^{+} \mathrm{A}^{-}\right)^{*}$ state can be formed via both routes, either in fluorescence quenching reaction [via exciplex formation, reaction (1)], or upon excitation within the weak CT absorption band of the ground-state-stable CT complex [scheme (3)]. And once again, as shown in Fig. 7, in liquid solution under photostationary conditions, CT fluorescence excited either via exciplex formation reaction or via direct excitation of $\mathrm{CT}$ complex is always the same, in accordance with the postulate formulated at an early stage of investigations of exciplex reaction [29] and in accordance with the observations of all other known exciplex/CT complexes studied so far. As mentioned earlier (cf. Sec. 1), the conclusion about

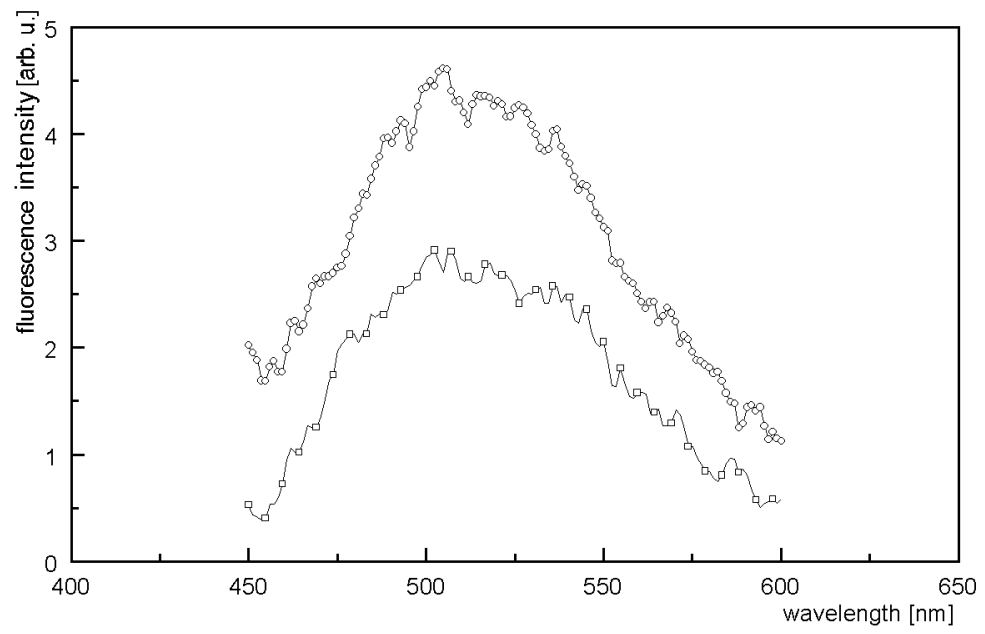

Fig. 7. Comparison of the fluorescence bands of naphthalene-TRCNE excited: in absorption band of naphthalene at $298 \mathrm{~nm}$ (circles), and CT absorption band of CT complex at $390 \mathrm{~nm}$ (squares).

the formation of weak CT complex between naphthalene and TRCNE is also in accordance with the results of investigations of association equilibria of TRCNE with various electron donors, according to which naphthalene-TRCNE system, in $\mathrm{CCl}_{4}$ solution at $20^{\circ} \mathrm{C}$, is characterized by the very small $\left(K_{\text {as }} \sim 3\right.$ ) association constant [27]. We should mention, for the record, that in dichloethane solution the excitation spectrum of exciplex fluorescence does not contain any additional CT absorption band, which is also consistent with the expectation (cf. Sec. 1) that in such polar solvent excitation of the complex results in an efficient formation of separated (solvated) ions of the donor and acceptor.

The presence of the ground-state-stable CT complex may indicate that the process of formation of naphthalene-TRCNE exciplex should not be considered as a purely dynamical one. However, there is no overlap between the fluores- 
cence band of naphthalene and that of exciplex, and/or of CT complex (an energy shift of their maxima is $\geq 10000 \mathrm{~cm}^{-1}$, cf. Fig. 3), which thus could obscure the analysis pertinent to the photokinetic scheme (4). Furthermore, the ground-state association of the donor and acceptor, which could introduce the problem of static quenching (cf. discussion by the end of Sec. 2) can probably be excluded in view of the small association constant, and this is further verified by the linear dependence of quenching of naphthalene fluorescence on the concentration of the quencher (cf. Sec. 4.2). Hence the naphthalene-TRCNE system can quite safely be treated as described by the photokinetic scheme (4). With this assumption, kinetics of the formation and relaxation of exciplex can be investigated by the analysis of monomer-exciplex equilibria under steady-state (photostationary) and time-resolved (transient) excitation conditions (cf. Sec. 2). The results of such analysis are presented and discussed throughout the following sections.

To this end, however, we should mention that investigations of excitation spectra of exciplex fluorescence for two other systems, i.e. for $p$-xylene-TRCNE and hexamethylbenzene-TRCNE, have brought about basically the same conclusions as in the above-described case of naphthalene-TRCNE system. But, in the case of $p$-xylene-TRCNE excitation spectrum of exciplex fluorescence shows that the CT absorption band is strongly overlapping with the molecular absorption of the donor. Very weak CT absorption can be traced (as an unusually wide and presumably multiple-band absorption) on a long-wavelength wing of the absorption band of $p$-xylene, and only under conditions of a very high concentration of $p$-xylene in solution - this automatically excludes the possibility of performance of any reliable quantitative analysis of fluorescence quenching reaction for $p$-xylene by TRCNE under photostationary conditions. On the other hand, its transient analysis was not possible for the same reason as explained below for hexamethylbenzene-TRCNE system.

In the case of hexamethylbenzene-TRCNE system the excitation spectrum of exciplex also reveals the presence of CT absorption. A wide and structureless CT absorption band has a maximum at $400 \pm 10 \mathrm{~nm}$, and is even more separated from the absorption band of the donor as compared with the case of naphthalene-TRCNE system. According to the findings cited above its CT complex (in $\mathrm{CCl}_{4}$ solution at $20^{\circ} \mathrm{C}$ ) is twice as strong as CT complex of naphthalene-TRCNE [27]. However, due to the experimental conditions of the present experiment we were not able to carry out the transient analysis of this system, which requires laser pulses of at least $275 \mathrm{~nm}$ wavelength - far outside the available range of the tuning (285-310 nm) of our double-jet dye laser. The results of analysis for monomer-exciplex equilibria for hexamethylbenzene-TRCNE system will be presented and discussed in the next papers of this series. In this paper we limit ourselves to the presentation and detailed discussion of monomer-exciplex equilibria for naphtalene-tricyanoethylene (NP-TRCNE) system. 


\subsection{Analysis of monomer-exciplex equilibrium of NP-TRCNE system under photostationary conditions}

We start our analysis of exciplex formation reaction between NP and TRCNE with checking the Stern-Volmer quenching law [28]:

$$
\left(I^{0} / I\right)=1+k_{\mathrm{q}} \tau_{0}[\mathrm{~A}]=1+K_{\mathrm{SV}}[\mathrm{A}]
$$

where $I$ and $I^{0}$ are the intensities of fluorescence of primarily excited molecule (NP molecule in this study) in the presence of quencher (TRCNE) in solution at a molar concentration $[\mathrm{A}]$, and when its concentration is $[\mathrm{A}]=0$, respectively; $k_{\mathrm{q}}$ - the quenching rate constant; $\tau_{0}$ - the fluorescence decay time of unquenched molecule; $K_{\mathrm{SV}}$ - the Stern-Volmer constant.

The plot of $\left\{\left(I^{0} / I\right)-1\right\}$ vs. concentration [A], given in Fig. 8, is linear in the whole studied concentration range of the quencher (and this observation excludes the contribution from the static quenching). It yields the Stern-Volmer constant for quenching NP fluorescence by TRCNE (in $n$-hexane solution at room temperature) of the value $K_{\mathrm{SV}}=4.7 \times 10^{3} \mathrm{M}^{-1}$. (As we will see in Sec. 4.4, this high value of the Stern-Volmer constant, remains in very satisfactory agreement with the corresponding $K_{\mathrm{SV}}$ value, determined from the transient analysis of monomer-exciplex equilibrium, $K_{\mathrm{SV}(\mathrm{tr})}=4.1 \times 10^{3} \mathrm{M}^{-1}$.) The decay time of unquenched fluorescence of NP (in $n$-hexane solution at room temperature) measured in this experiment, was found to be $\tau_{0}=63.8 \mathrm{~ns}$. And this leads to the value of the quenching rate constant $k_{\mathrm{q}}=\left(K_{\mathrm{SV}} / \tau_{0}\right)=7.36 \times 10^{10} \mathrm{M}^{-1} \mathrm{~s}^{-1}$. The quenching rate constant $k_{\mathrm{q}}$ shows only a slight temperature dependence (in the range of temperatures limited by the boiling point of $n$-hexane), as shown by the data of Table I.

According to the results of the Stern-Volmer analysis, TRCNE must be considered as a very strong quencher of excited NP in non-polar liquid solutions. Its interaction with excited donor leads effectively to the formation of $\left(\mathrm{D}^{+} \mathrm{A}^{-}\right)^{*}$, NP-TRCNE exciplex. As we mentioned in Sec. 1, there were earlier reports on exciplex formation between a series of polycyclic aromatic hydrocarbon donors and trans-1,2-dicyanoethylene (DCNE) - another electron acceptor from the cyanoethylenes family [25]. In this cited work, all characteristics pertinent to the fluorescence quenching reaction with the formation of exciplex have been observed in a series of solvents of different polarities, from benzene $(\varepsilon=2.27)$ to dichloroetane $(\varepsilon=8.90)$. The Stern-Volmer quenching has also been analyzed, however, in the case of NP-DCNE system (NP was the donor of the highest ionization potential in the investigated series) the Stern-Volmer constant has not been determined. For the sake of comparison with the present results we have performed additional measurements for NP-DCNE system in $n$-hexane solution. Their results in general conform to earlier observations reported in [25]. We have determined the Stern-Volmer constant $K_{\mathrm{SV}}$ which in this case has a value of $1.39 \times 10^{3} \mathrm{M}^{-1}$ (and the corresponding value of the quenching rate constant is $k_{\mathrm{q}}=3.5 \times 10^{10} \mathrm{M}^{-1} \mathrm{~s}^{-1}$ ). Data illustrating the temperature dependence of $k_{\mathrm{q}}$ for NP-DCNB system are also collected in Table I for comparison. 


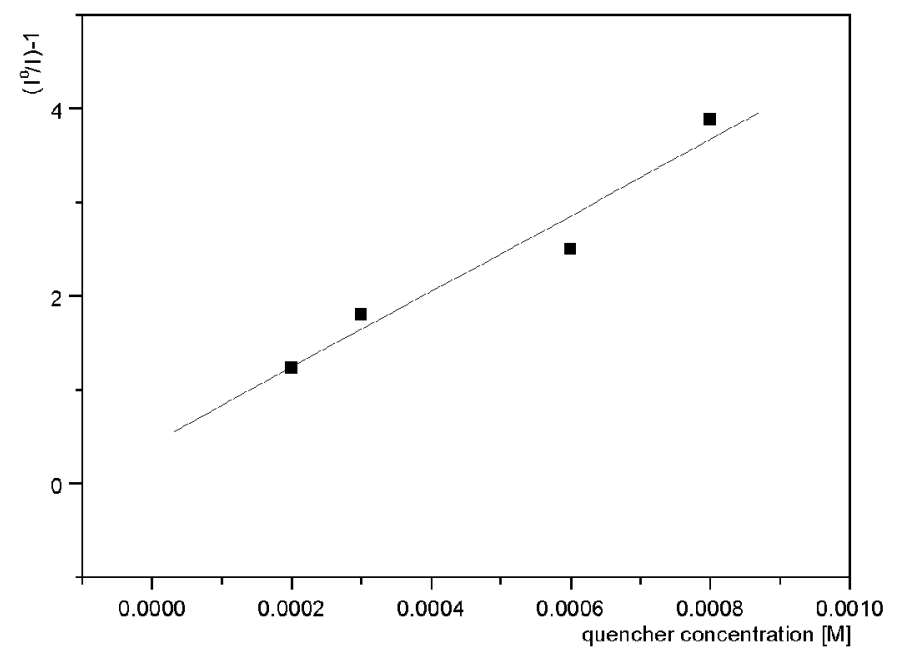

Fig. 8. Stern-Volmer plot for quenching naphthalene fluorescence by TRCNE in $n$-hexane solution at room temperature.

\section{TABLE I}

Temperature changes of the quenching rate constant $k_{\mathrm{q}}$ of naphthalene (monomer) fluorescence by tricyanoethylene (TRCNE) and trans-1,2-dicyanoethylene (DCNE) electron acceptors, in $n$-hexane solution.

\begin{tabular}{c|c|c}
\hline \hline $\begin{array}{c}\text { Temperature } \\
T(\mathrm{~K})\end{array}$ & $\begin{array}{c}\text { TRCNE } \\
k_{\mathrm{q}}\left(10^{10} \mathrm{M}^{-1} \mathrm{~s}^{-1}\right)\end{array}$ & $\begin{array}{c}\text { DCNE } \\
k_{\mathrm{q}}\left(10^{10} \mathrm{M}^{-1} \mathrm{~s}^{-1}\right)\end{array}$ \\
\hline 298 & 7.36 & 3.50 \\
318 & 9.00 & 3.10 \\
328 & 9.30 & 2.20
\end{tabular}

If we recall that in liquid non-polar solutions tetracyanoethylne (TCNE), which is the strongest electron acceptor in cyanoethylene series, forms firmly strong ground-state-stable EDA complexes [14] with very distinct CT absorption bands, and at the same time completely quenches donors' fluorescence with neither traces of exciplex fluorescence, nor CT fluorescence (directly excited in CT absorption band), then we arrive at the conclusion that the observed efficiency of quenching of a given excited donor molecule by electron acceptors from the series of cyanoethylenes family follows their electron-accepting ability (electron affinity). This of course does not mean that one should expect that the kinetics of the formation and relaxation of their exciplexes (even with the same given donor) would change in a similar manner, as the kinetics (and dynamics) is controlled and governed by the "fragile" balance and interplay between the rate constants (and by their 
different sensitivity to environmental conditions) of all the processes operating within the photokinetic scheme (4). This is very clearly demonstrated by the fact of completely reversed temperature dependence of the quenching rate constant $k_{\mathrm{q}}$ for both systems under consideration. Table I shows that, under the same experimental conditions, $k_{\mathrm{q}}$ for NP-TCNE is increasing with increasing temperature, while $k_{\mathrm{q}}$ for NP-DCNE system displays an opposite direction of changes.

Kinetic coupling between the emissions of excited monomer $\left(D^{*}\right)$ and exciplex, $\left(\mathrm{D}^{+} \mathrm{A}^{-}\right)^{*}$, species within the framework of the photokinetic scheme (4), provides several routes of investigations of the sensitivity of exciplex formation and relaxation processes to the environmental conditions (temperature, polarity, and viscosity of solvents, etc.). Under photostationary conditions, i.e. when the excitation function is time-independent [i.e. $I_{a}(t)=$ const in Eq. (5)], the steady-state analysis of the population equations (5) provides the following expression for the exciplex-to-monomer intensity ratio:

$$
\frac{I_{\mathrm{E}}}{I_{\mathrm{M}}[\mathrm{A}]}=\frac{k_{5}}{k_{1}} \frac{k_{3}[\mathrm{~A}]}{k_{3}+k_{\mathrm{p}}}
$$

where $I_{\mathrm{E}}$ and $I_{\mathrm{M}}$ are integrated intensities of fluorescence bands of the exciplex and monomer (donor), respectively [all the others kinetic parameters have their meaning as defined in the photokinetic scheme (4)]. The temperature dependence of $I_{\mathrm{E}} / I_{\mathrm{M}}$ ratio is mainly controlled by the rates of exciplex formation $\left(k_{3}\right)$ and its thermal (feedback) dissociation $\left(k_{4}\right)$, which are usually the most temperature-sensitive processes of the reaction (4). The Arrhenius-type plot of $\ln \left(I_{\mathrm{E}} / I_{\mathrm{M}}\right)$ vs. $(1 / T)$, known as the Stevens-Ban plot $[30]$ is particularly useful, as it allows us to determine fundamental thermodynamics parameters (activation energies of exciplex formation and its feedback dissociation and the heat of the formation of an exciplex). The Stevens-Ban plot has two limits set by the competition between the thermal dissociation $\left(k_{4}\right)$ and the decay of exciplex $\left(k_{\mathrm{p}}\right)$. These limits are reached either when $k_{4} \gg k_{\mathrm{p}}$ (known as high temperature limit - HTL), or when $k_{4} \ll k_{\mathrm{p}}$ (low temperature limit - LTL). Under LTL conditions the slope of the Stevens-Ban plot yields the value of $E_{3}-$ an activation energy for exciplex formation process. Under HTL conditions the slope of the Stevens-Ban plot yields the value of the heat of the formation of exciplex, $-\Delta H=\left(E_{4}-E_{3}\right)$, and thus a combination of both, the results of HTL and LTL, yields also the value of activation energy for exciplex thermal dissociation, $E_{4}$.

Figure 9 shows the Stevens-Ban plot for NP-TRCNE monomer-exciplex equilibrium in $n$-hexane solution. It is seen that under conditions of the present experiment, exciplex-to-monomer fluorescence intensity ratio exhibit only LTL behavior $\left(k_{4} \ll k_{\mathrm{p}}\right)$, from which one can extract only an activation energy, $E_{3}$.

The fact that the Stevens-Ban plot for NP-TRCNE monomer-exciplex equilibrium follows, in $n$-hexane solution only a low-temperature limit, indicates that the formation of exciplex is controlled by the rate constant of diffusion-controlled fluorescence quenching, i.e. $k_{3} \approx k_{\mathrm{q}}$, whereas its relaxation is practically governed 


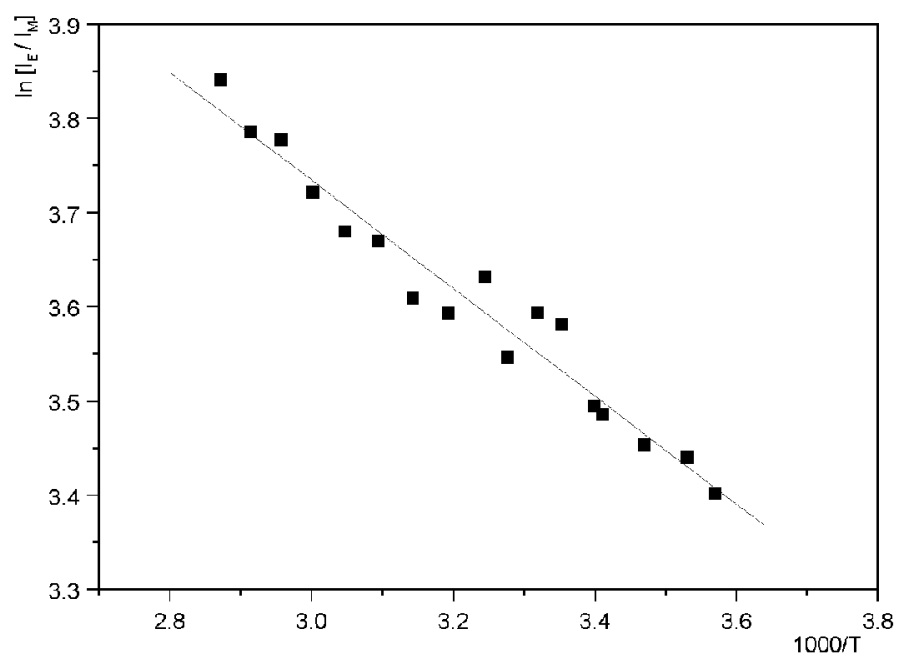

Fig. 9. Stevens-Ban plot for naphthalene-TRCNE system in $n$-hexane solution.

by its decay $k_{\mathrm{p}}\left(=k_{5}+k_{6}\right)$. However, such conclusions based on observations for LTL only may be of a very limited accuracy, unless the temperature dependence of radiative rate constants of exciplex $\left(k_{5}\right)$ and monomer $\left(k_{1}\right)$, as well as of $k_{3}$, is determined. On the other hand, an activation energy for NP-TRCNE exciplex formation, extracted from the slope of the Stevens-Ban plot in Fig. 9, is $E_{3}=4.8 \mathrm{~kJ} \cdot \mathrm{M}^{-1}$. Such a very small value for the energy of exciplex formation (at least two or three times smaller than for typical exciplex systems [31]) might indicate that there is no energy barrier for NP-TRCNE exciplex formation in such a solvent as $n$-hexane (may be except for a viscous flow). However, this system is not an exceptional one, for instance very low energy of exciplex formation has already been observed for dicyanobenzene-toluene exciplex [32]). We will discuss possible causes, which make the formation process of NP-TRCNE exciplex so effective, later on.

\subsection{Time-resolved fluorescence spectra of NP-TRCNE system}

Time-resolved fluorescence spectra of NP-TRCNE system, shown in Fig. 10, are directly confirming an exciplex mechanism of bimolecular fluorescence quenching with a formation of $\left(\mathrm{D}^{+} \mathrm{A}^{-}\right)^{*}$ excited CT state. Like in the case of steady-state fluorescence spectra (cf. Fig. 3), also time-resolved spectra consist of two different and spectrally well-separated fluorescence bands, a monomer (primarily excited NP molecular fluorescence) and an exciplex fluorescence band. Both, monomer and exciplex fluorescence bands, do not undergo any spectral changes (band position and shape) during their time evolution, but the ratio of their intensities, $I_{\mathrm{E}} / I_{\mathrm{M}}$, undergoes changes (or variations) for different delay times of their registration (measured from an initial excitation pulse). With the time elapse, intensity 


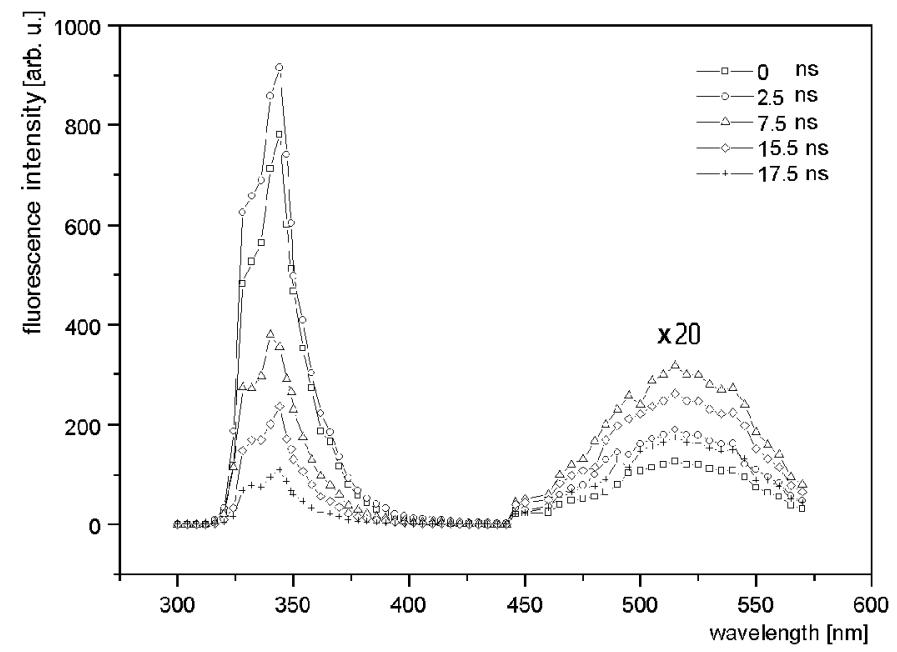

Fig. 10. Time-resolved fluorescence spectra of naphthalene-TRCNE EDA system in $n$-hexane solution at room temperature recorded after different delay times as indicated in top right corner.

of monomer fluorescence band, $I_{\mathrm{M}}$, is continuously decreasing, while the intensity of exciplex fluorescence band, $I_{\mathrm{E}}$, is increasing during the first steps of observations and reaches its maximum after $7.5 \div 10$ ns time period, after which it is continuously decreasing. These qualitative observations seem to indicate that the photokinetic scheme (4) is applicable for the description of the transient analysis of monomer-exciplex equilibrium under consideration.

\subsection{Transient analysis of monomer-exciplex equilibrium of NP-TRCNE system}

We assume that the time-dependent excitation function $I_{a}(t)$ is of $\delta$-pulse type (which is a safe assumption for short-pulsed laser excitation used in our experiments), and that at time $t=0$ it produces an initial concentration $\left[\mathrm{D}^{*}\right]_{0}$ of excited monomer (while at the same time, $t=0$, the concentration of exciplex equals 0 ). With these assumptions the population equations (5) can be combined and quite easily solved, yielding the well-known solutions which describe temporal evolution of intensity of monomer and exciplex fluorescence (or their so-called fluorescence response functions) [28]:

$$
\begin{aligned}
& I_{\mathrm{M}}(t)=k_{1} \frac{\lambda_{2}-X}{\lambda_{2}-\lambda_{1}}\left[\exp \left(-\lambda_{1} t\right)+A \exp \left(-\lambda_{2} t\right)\right], \\
& I_{\mathrm{E}}(t)=\frac{k_{5} k_{3}[\mathrm{~A}]}{\lambda_{2}-\lambda_{1}}\left[\exp \left(-\lambda_{1} t\right)-\exp \left(-\lambda_{2} t\right)\right],
\end{aligned}
$$

where the decay parameters $\lambda_{1}$ and $\lambda_{2}$ are defined as: 


$$
\lambda_{1,2}=(1 / 2)\left[X+Y \mp\left\{(Y-X)^{2}+4 k_{4} k_{3}[\mathrm{~A}]\right\}^{1 / 2}\right]
$$

and supplementary parameters (introduced in order to simplify the analysis) were defined as:

$$
\begin{aligned}
& X=k_{0}+k_{3}[\mathrm{~A}], \\
& Y=k_{\mathrm{p}}+\mathrm{k}_{4}, \\
& A=\left(X-\lambda_{1}\right) /\left(\lambda_{2}-X\right) .
\end{aligned}
$$

Equations (8) and (9) predict two-component decay curves (or fluorescence time profiles) for the monomer and exciplex fluorescence. It is a double-exponential decay curve for monomer (as the concentration of initially excited monomer molecules $\left[\mathrm{D}^{*}\right]_{0}$ decreases via its internal decay channels $\left(k_{0}\right)$ — "unquenched" monomer molecules, as well as via exciplex formation $\left(k_{3}[\mathrm{~A}]\right)$ — "quenched" monomer molecules). The decay curve for exciplex fluorescence comprises, however, the rise component and the decay component. We note that the amplitudes (preexponential factors) of both decay parameters in Eq. (9) are equal and of opposite sign (their ratio equals -1 ), and hence the rise component of exciplex fluorescence (a growing-in of exciplex fluorescence) is always described by $\lambda_{2}$ decay parameter (of negative amplitude, as the exciplex concentration, $\left[\left(\mathrm{D}^{+} \mathrm{A}^{-}\right)^{*}\right]$, can never be negative), which is the fastest process, since $\lambda_{2}>\lambda_{1}$ according to Eq. (10). Formally, one might also expect two sets of identical decay parameters for monomer and exciplex fluorescence decay curves (at least, as long as there is no overlap between monomer and exciplex emissions, and if the formation of the ground-state stable CT complex can be neglected, which is the case of NP-TRCNE system, cf. discussions in Secs. 2 and 4.1).

Typical decay curves (time profiles) of monomer and exciplex fluorescence of NP-TRCNE system, in $n$-hexane solution, for two different quencher concentrations and at different temperatures, are given in Figs. 11 and 12. The decay parameters $\lambda_{1}$ and $\lambda_{2}$, retrieved from all measured decay curves are collected in Table II (cf. also captions to Figs. 11 and 12). They show quantitative differences under different experimental conditions (concentration of quencher [A] and temperature), but general characteristics of the observed decays and their relationship remain always the same, although they do not comply fully with the expectations outlined by Eqs. (8) and (9).First of all, the observed decay curves of monomer fluorescence are always single-exponential. The decay curves of exciplex fluorescence, as expected, show the rise component (exciplex growing-in) and the decay component, however, the rise component is much shorter than single-exponential monomer decay (for any given concentration of the acceptor $[\mathrm{A}]$ ), which in turn seems to correlate rather to the decay of exciplex fluorescence.

The observation of strictly single-exponential decay of monomer fluorescence indicates that feedback dissociation of exciplex $\left(k_{4}\right)$ is negligible, which is in agreement with the earlier conclusion, which we arrived at in the course of the 

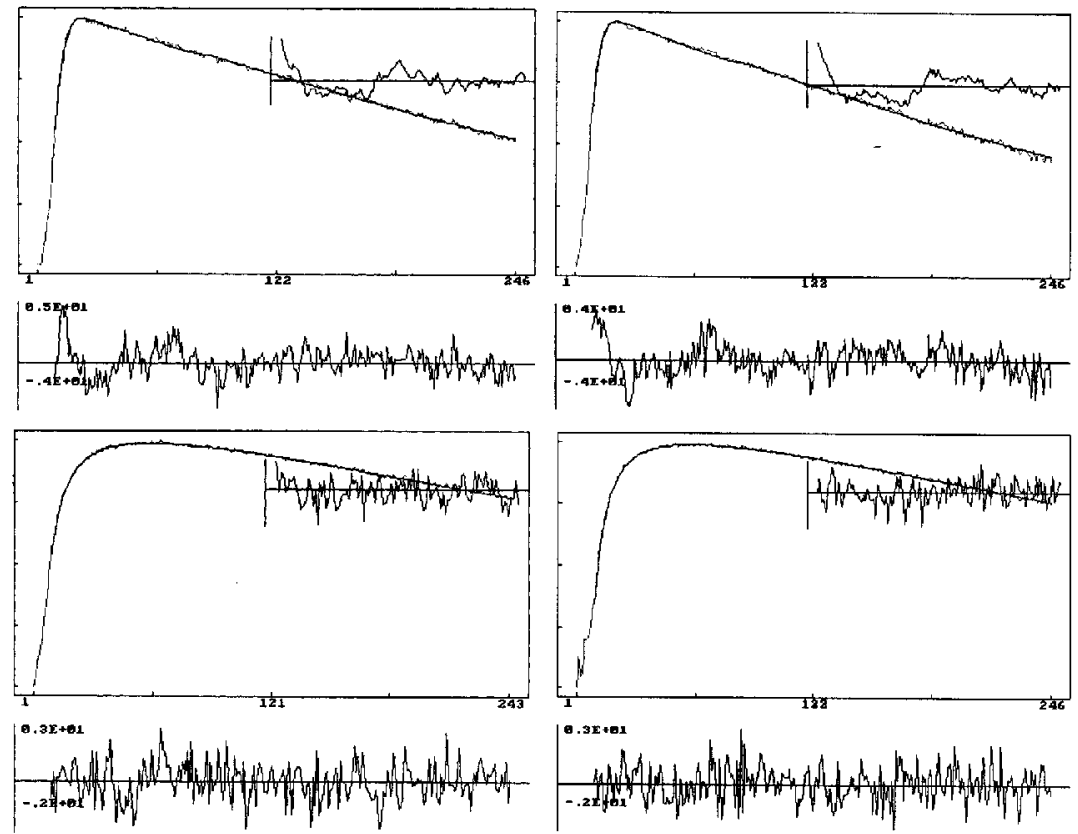

Fig. 11. Decay curves of the monomer and exciplex fluorescence of naphthaleneTRCNE EDA system in $n$-hexane solution monitored for maximum of monomer (napthalene) fluorescence at $330 \mathrm{~nm}$ (top) and for maximum of exciplex fluorescence at $520 \mathrm{~nm}$ (bottom) at the temperature of: $298 \mathrm{~K}$ (left panel) and $323 \mathrm{~K}$ (right panel). Concentration of NP is $4 \times 10^{-3} \mathrm{M}$ and that of TRCNE $-6 \times 10^{-4} \mathrm{M}$. Fluorescence excitation within the naphthalene absorption band at $298 \mathrm{~nm}$. Intensity scale on the abscissas is logarithmic; the ordinates are scaled $0.155 \mathrm{~ns}$ per channel. Decay times $\left(\tau_{i}=1 / \lambda_{i}\right.$, cf. Eqs. (8-13)), determined from the analysis are: (top left) $\tau_{1}^{\mathrm{M}}=6.11 \mathrm{~ns}$; (bottom left) $\tau_{1}^{\mathrm{E}}=20.17 \mathrm{~ns}, \tau_{2}^{\mathrm{E}}=3.61 \mathrm{~ns}$ (with amplitudes ratio $A_{1}^{\mathrm{E}} / A_{2}^{\mathrm{E}}=-1.03$ ); (top right) $\tau_{1}^{\mathrm{M}}=14.40 \mathrm{~ns}$; (bottom right) $\tau_{1}^{\mathrm{E}}=17.82 \mathrm{~ns}, \tau_{2}^{\mathrm{E}}=3.56 \mathrm{~ns}$ (with amplitudes ratio $\left.A_{1}^{\mathrm{E}} / A_{2}^{\mathrm{E}}=-1.02\right)$.

steady-state analysis (cf. Sec. 4.2). If one assumes that $k_{\mathrm{p}} \gg k_{4} \approx 0$, then from the inspection of Eqs. (10) through (13), following the limits for decay parameters and their amplitudes it can be found:

$$
\begin{aligned}
& \lambda_{1} \rightarrow X=k_{0}+k_{3}[\mathrm{~A}] \text { (with ultimate limit) } \lambda_{1} \rightarrow k_{0}, \text { when } k_{3}[\mathrm{~A}] \rightarrow 0 \text { ), } \\
& \lambda_{2} \rightarrow Y=k_{\mathrm{p}} \\
& A \rightarrow 0 .
\end{aligned}
$$

It immediately comes from the inspection of Eqs. (8) and (9) that under such limits: 

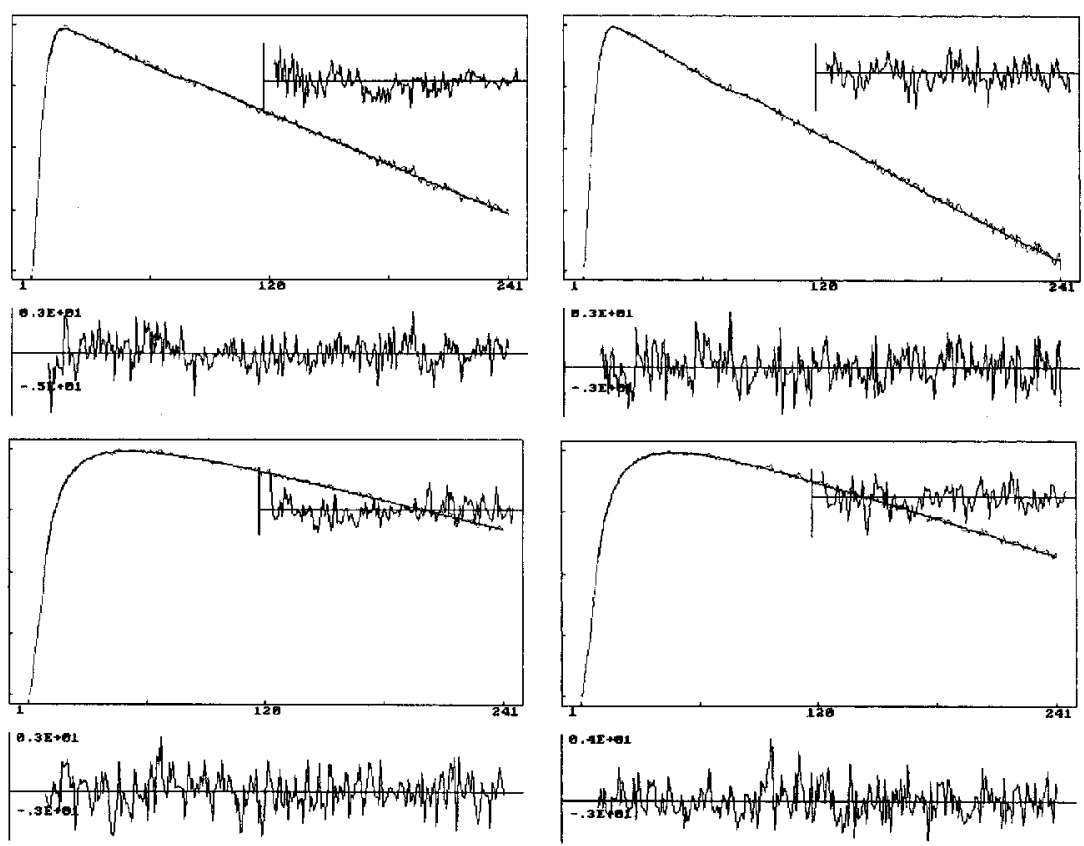

Fig. 12. Decay curves of the monomer and exciplex fluorescence of naphthaleneTRCNE EDA system in $n$-hexane solution monitored for maximum of monomer (napthalene) fluorescence at $330 \mathrm{~nm}$ (top) and for maximum of exciplex fluorescence at $520 \mathrm{~nm}$ (bottom) at the temperature of: $298 \mathrm{~K}$ (left panel) and $323 \mathrm{~K}$ (right panel). Concentration of NP is $4 \times 10^{-3} \mathrm{M}$ and that of TRCNE $-8 \times 10^{-4} \mathrm{M}$. Fluorescence excitation within the naphthalene absorption band at $298 \mathrm{~nm}$. Intensity scale on the abscissas is logarithmic; the ordinates are scaled $0.155 \mathrm{~ns}$ per channel. Decay times $\left(\tau_{i}=1 / \lambda_{i}\right.$, cf. Eqs. (8-13)), determined from analysis are: (top left) $\tau_{1}^{\mathrm{M}}=15.1 \mathrm{~ns}$; (bottom left) $\tau_{1}^{\mathrm{E}}=15.43 \mathrm{~ns}, \tau_{2}^{\mathrm{E}}=3.32 \mathrm{~ns}$ (with amplitudes ratio $A_{1}^{\mathrm{E}} / A_{2}^{\mathrm{E}}=-0.98$ ); (top right) $\tau_{1}^{\mathrm{M}}=12.54 \mathrm{~ns} ;$ (bottom right) $\tau_{1}^{\mathrm{E}}=14.0 \mathrm{~ns}, \tau_{2}^{\mathrm{E}}=3.18 \mathrm{~ns}$ (with amplitudes ratio $\left.A_{1}^{\mathrm{E}} / A_{2}^{\mathrm{E}}=-1.04\right)$.

- The decay of monomer fluorescence must become strictly single-exponential (as for $A=0$ (cf. (16)) the second exponent term in Eq. (8) is vanishing). Thus, the decay parameter of monomer fluorescence would be $\lambda_{1}$, which according to (14) is determined by the exciplex formation process (it should reach the value of $k_{0}$ rate constant of monomer decay, when the exciplex formation process becomes negligible), and one should expect that the observed decay time of monomer fluorescence should become shorter upon the increase of quencher concentration [A]. However, it is important to realize that even when the formation process of exciplex is all the time effective, the decay of monomer fluorescence would be strictly single-exponential; 
TABLE II

Temperature dependence of decay times of monomer $\left(\tau_{1}^{\mathrm{M}}\right)$ and exciplex $\left(\tau_{1}^{\mathrm{E}}, \tau_{2}^{\mathrm{E}}\right)$ fluorescence of naphthalene-tricyanoethylene system in $n$-hexane solutions containing different concentrations [TRCNE] of the quencher (concentration of naphthalene is constant, $[\mathrm{NP}]=$ $4 \times 10^{-3} \mathrm{M}$ )

\begin{tabular}{c|c|c|c||c|c|c}
\hline \hline & \multicolumn{3}{|c||}{$[\mathrm{TRCNE}]=3 \times 10^{-4} \mathrm{M}$} & \multicolumn{3}{c}{$[\mathrm{TRCNE}]=8 \times 10^{-4} \mathrm{M}$} \\
\hline$T(\mathrm{~K})$ & $\tau_{1}^{\mathrm{M}}(\mathrm{ns})$ & $\tau_{1}^{\mathrm{E}}(\mathrm{ns})$ & $\tau_{2}^{\mathrm{E}}(\mathrm{ns})$ & $\tau_{1}^{\mathrm{M}}(\mathrm{ns})$ & $\tau_{1}^{\mathrm{E}}(\mathrm{ns})$ & $\tau_{2}^{\mathrm{E}}(\mathrm{ns})$ \\
\hline 298 & 30.70 & 27.23 & 3.37 & 15.09 & 15.43 & 3.32 \\
303 & 29.11 & 18.61 & 3.80 & 14.15 & 15.54 & 3.27 \\
313 & 25.86 & 16.88 & 3.92 & 13.44 & 14.09 & 3.22 \\
323 & 24.48 & 15.92 & 3.86 & 12.54 & 14.05 & 3.19 \\
\hline \multicolumn{6}{|c|}{$[\mathrm{TRCNE}]=6 \times 10^{-4} \mathrm{M}$} & \multicolumn{3}{c}{$[\mathrm{TRNE}]=3.0 \times 10^{-3} \mathrm{M}$} \\
\hline$T(\mathrm{~K})$ & $\tau_{1}^{\mathrm{M}}(\mathrm{ns})$ & $\tau_{1}^{\mathrm{E}}(\mathrm{ns})$ & $\tau_{2}^{\mathrm{E}}(\mathrm{ns})$ & $\tau_{1}^{\mathrm{M}}(\mathrm{ns})$ & $\tau_{1}^{\mathrm{E}}(\mathrm{ns})$ & $\tau_{2}^{\mathrm{E}}(\mathrm{ns})$ \\
\hline 298 & 16.11 & 20.17 & 3.61 & 8.03 & 7.90 & 2.87 \\
303 & 16.00 & 20.41 & 3.59 & 7.63 & 7.60 & 2.84 \\
313 & 14.99 & 18.64 & 3.57 & 7.16 & 7.10 & 2.83 \\
323 & 14.40 & 17.82 & 3.57 & 6.76 & 6.83 & 2.70
\end{tabular}

- The decay curve of exciplex, as described by Eq. (9), still remains double-exponential. Its decay component would be determined by $\lambda_{1}$ decay parameter, which, as in the case discussed just above, is determined by the exciplex formation process. It should also approach an ultimate limit of $k_{0}$ rate constant of monomer decay and should be dependent on quencher concentration $[\mathrm{A}]$. We note that both, the decay of monomer and of exciplex fluorescence, are expected to correlate one to each other;

- The rise component of exciplex fluorescence (as mentioned earlier, always determined by the $\lambda_{2}$ decay parameter) would correspond to the rate constant of exciplex fluorescence decay $k_{\mathrm{p}}$ (cf. (15)) - the observed rise time of exciplex fluorescence is expected to be independent on the quencher concentration $[\mathrm{A}]$. It is important to stress that, contrary to common expectations, the rise (growing-in) of exciplex fluorescence is not connected to the decay of monomer fluorescence, and thus it would not contain any information on the exciplex formation, instead it would be determined by the exciplex decay time $1 / k_{\mathrm{p}}$ (this implies that $k_{\mathrm{p}}>\mathrm{k}_{0}$, as the rate constant for exciplex feedback dissociation $k_{4}=0$ ).

Let us compare now, once again, experimental decay curves (Figs. 11, 12 and Table II) with the above predictions. Briefly, we can summarize experimental observations as follows:

- For the whole range of investigated concentrations (of TRCNE) and of tem- 
peratures, the decays of monomer fluorescence are always single-exponential, while the decay curves of exciplex fluorescence contain both, the rise and the decay components.

- The observed decays of monomer fluorescence and of exciplex fluorescence, although not strictly parallel, are always close one to each other (within $\pm 10 \%$ difference for the values of the decay times, and only for the lowest concentration of TRCNE they differ by more than $\pm 20 \%$ ). Both of them are showing a quite remarkable shortening with increasing concentration of TRCNE (by the factor of 4 for the change of concentration by one order of magnitude). But even at the lowest concentration of TRCNE they are still far from the limit set by the rate constant of monomer decay $k_{0}$ (i.e. $k_{0}=$ $1 / \tau_{0}=1.57 \times 10^{7} \mathrm{~s}^{-1}$ for "unquenched" naphthalene fluorescence at $298 \mathrm{~K}$; cf. Table III). They also show temperature dependence which is getting less pronounced with an increase in the TRCNE concentration (almost negligible at the highest concentration, cf. Table II).

- The observed growing-in (the rise component) of exciplex fluorescence has the rise time which is always much shorter than the decay time of monomer and/or exciplex fluorescence. Its value of $3.6 \pm 0.3 \mathrm{~ns}$ does not depend on the concentration of TRCNE and only for the highest concentration it becomes clearly shorter. Upon an increase in temperature the rise time is very moderately shortening (cf. Table II).

TABLE III

Temperature dependence of kinetic rate constants of NP-TRCNE system and the activation energy of NP-TRCNE exciplex formation $\left(E_{3}\right)$.

\begin{tabular}{c|c|c|c|c}
\hline \hline$T$ & $k_{0}$ & $k_{3}$ & $k_{\mathrm{p}}$ \\
$(\mathrm{K})$ & $\left(10^{7} \mathrm{~s}^{-1}\right)$ & $\left(10^{10} \mathrm{M}^{-1} \mathrm{~s}^{-1}\right)$ & $\begin{array}{c}E_{3} \\
\left(10^{8} \mathrm{~s}^{-1}\right)\end{array}$ & $(\mathrm{kJ} / \mathrm{M})$ \\
\hline 298 & 1.57 & 6.38 & 2.76 & - \\
303 & 1.71 & 7.32 & 2.85 & - \\
313 & 1.80 & 7.43 & 2.80 & - \\
323 & 1.82 & 7.92 & 2.82 & - \\
- & - & - & - & 5.4
\end{tabular}

In view of these observations we may safely conclude that, in principle the decay curves for both, the monomer and exciplex fluorescence of NP-TRCNE system, are in quite satisfactory agreement with earlier-discussed predictions, derived from Eqs. (8) through (13) under conditions set by limits (14) through (16). Hence, the analysis of the kinetics of monomer-exciplex equilibrium of NP-TRCNE can be performed within the framework of the photokinetic scheme (4). At this point, 
however, we should stress the fact that under conditions of the present experiment, NP-TRCNE system presents itself as a very interesting although peculiar case. As was mentioned earlier in this Section, the rise (growing-in) of exciplex fluorescence is determined by the exciplex decay time, and as such does not carry any information on the exciplex formation process (contrary to the prevailing majority of studied exciplexes). For such an information one should address the decay of fluorescence of the monomer (and/or of exciplex). Therefore, we are dealing with the case where common physical meaning of the decay parameters $\lambda_{1}$ and $\lambda_{2}$ is reversed. Such kinetics of formation and realization is rarely displayed by (or observed for) exciplexes, though it has been clearly demonstrated and discussed in the case of molecular excimers [33].

Once, the decay parameters were retrieved, with the use of double-exponential-fitting procedures (cf. Sec. 3), from the observed time profiles of monomer and exciplex fluorescence, one can try to determine the rate constants of other individual processes involved in photokinetic scheme (4), applicable to the present case of NP-TRCNE system. In order to execute this task, one can follow anyone of the well-known and checked recipes (see for instance $[28,31,32]$ ). In the case under consideration, the following relations between decay parameters and the rate constants are valid (according to relations (10) through (16)):

$$
\begin{aligned}
& \lambda_{1}+\lambda_{2}=k_{3}[\mathrm{~A}]+k_{0}+k_{\mathrm{p}}, \\
& \lambda_{1} \cdot \lambda_{2}=k_{\mathrm{p}} k_{3}[\mathrm{~A}]+k_{0} k_{\mathrm{p}},
\end{aligned}
$$

and from their concentration (of the quencher, TRCNE) and temperature dependence, all other (e.g. $k_{3}$ and $k_{\mathrm{p}}$ ) rate constants of individual processes can be found ( $k_{0}$ - the rate constant of the decay of "unquenched" naphthalene molecule, being accessible from direct measurements). With the assumption of the Arrhenius-type activation of the exciplex formation process, its activation energy $E_{3}$ (as defined in Sec. 4.2) can also be determined from the temperature dependence of $k_{3}$ rate constant. The results of such analysis are collected in Table III.

The inspection of data in Table III shows that the rate constant of exciplex formation, $k_{3}$, undergoes the strongest temperature dependence among the rate constants determined, although its increase is rather moderate (by ca. 25\%) within the limited range of investigated temperatures $(25 \mathrm{~K})$. If we compare a value of $k_{3}$ and its temperature dependence with the relevant data gathered in the course of the photostationary analysis (cf. Sec. 4.2, and Table I) for the quenching rate constant $k_{\mathrm{q}}$, then we see that although these latter values are somewhat larger (in an acceptable 15\% range) at corresponding temperatures, the temperature dependence of $k_{\mathrm{q}}$ is accurately the same as the one observed in the course of the transient analysis. The transient analysis delivers also the quenching constant $K_{\mathrm{SV}(\mathrm{tr})}=k_{3} / k_{0}$ (analogous to the Stern-Volmer constant $K_{\mathrm{SV}}$ under photostationary conditions), and its value $K_{\mathrm{SV}(\mathrm{tr})}=4.07 \times 10^{3} \mathrm{M}^{-1}$ at $298 \mathrm{~K}$ is, within the error limits of analysis, in good agreement with $K_{\mathrm{SV}}$ value determined within the framework of photostationary analysis of the Stern-Volmer plots (cf. Sec. 4.2). 
An activation energy for exciplex formation, extracted from the temperature dependence of the $k_{3}$ rate constant, is $E_{3}=5.4 \mathrm{~kJ} / \mathrm{M}$ and very reasonably agrees with the value (of $4.8 \mathrm{~kJ} / \mathrm{M}$ ) delivered by the analysis of the Stevens-Ban plots for LTL limit (cf. Sec. 4.2). Once again it points out that, in practice, there is no energy barrier for the formation process of NP-TRCNE exciplex in $n$-hexane solution. With a very large rate constant of exciplex formation, even as for the diffusion-controlled process, one could speculate about additional factors and/or mechanisms that support the very efficient exciplex formation process (however, we probably should exclude any long-range mechanism of electron transfer from the donor to acceptor in such solvent of low polarity as $n$-hexane [34], although on the other hand one should bear in mind that TCNE - the strongest electron acceptor in cyanoethylenes family - quenches virtually every molecular emission, even in nonpolar solvents and without measurable traces of exciplex formation (as discussed in Sec. 1). This problem must remain open for further studies of NP-TRCNE system in different solvents.

The rate constant of monomer fluorescence decay $k_{0}$ was determined by direct measurements of decay times of ("unquenched") fluorescence of napthalene in $n$-hexane solutions at different temperatures, and with the assumption that naphthalene fluorescence quantum yield (which is $\Phi=0.23$ [35] at room temperature) does not change within the investigated temperature range. This may introduce an error in the estimations of the rate constant of exciplex fluorescence decay $k_{\mathrm{p}}$ (an "intrinsic" decay of excited exciplex). However, in view of the fact that at room temperature, at which both rate constants are determined "accurately", $k_{\mathrm{p}}$ is almost 20 times as large as $k_{0}$ (cf. Table III), we believe that the error introduced by the assumption of the temperature-independent (in a very narrow temperature range of $25^{\circ} \mathrm{C}$ above room temperature) quantum yield of naphthalene fluorescence, should not exceed limits of the accuracy of the transient analysis.

The rate constant of exciplex decay, $k_{\mathrm{p}}$, which for this particular exciplex determines its observed rise time was found to be, within the accuracy of the analysis, temperature-independent (cf. Table III). This, together with the virtual independence of $k_{\mathrm{p}}$ of the concentration of TRCNE, reasonably agrees with the lack of exciplex feedback dissociation $\left(k_{4} \approx 0\right)$, as inferred in the course of both, the photostationary and the transient analysis of monomer-exciplex equilibrium. It seems obvious now that only under such circumstances (and only in the low-temperature-limit), NP-TRCNE monomer-exciplex equilibrium could display kinetics with the reversed physical meaning of the decay parameters.

\section{Summary and concluding remarks}

The results of the measurements and analysis performed within this work has clearly shown that tricyanoethylene, new electron acceptor from the family of cyano-substituted ethylenes must be considered as a very strong electron acceptor. In photoinduced reactions with typical aromatic hydrocarbon electron donors 
( $p$-xylene, hexamethylbenzene, and naphthalene) tricyanoethylene acts as a very strong quencher of the fluorescence of donor molecules. Bimolecular fluorescence quenching reaction leads to the formation of exciplexes.

For naphthalene-tricyanoethylene exciplex, the detailed and complete analysis of monomer-exciplex equilibrium has been performed in $n$-hexane solution under photostationery and transient conditions, within the framework of a simple two-state photokinetic scheme, which has turned out to be applicable to this case with a very satisfactory accuracy. Most important observations and conclusions which come from the results of this analysis can be summarized as follows:

- the exciplex formation reaction in nonpolar solution of $n$-hexane is fully diffusion-controlled, barrierless reaction and there is no participation of exciplex feedback dissociation process in relaxation (depletion) channels of the exciplex,

- the observed kinetics of the monomer-exciplex equilibrium displays a very peculiar case of the reversed physical meaning of the decay parameters for exciplex fluorescence - the rise time (or growing-in) of exciplex emission is controlled by the exciplex decay time, while the exciplex formation process corresponds to the decay time of monomer fluorescence.

Although such kinetics of exciplex formation and relaxation is not commonly encountered, it by no means should be considered as a very unique case. The photoinduced reaction of exciplex formation is always governed and controlled by the rate constants (first and second-order) of all radiative and nonradiative processes pertinent to a given electron-donor-acceptor system under a particular set of environemental (experimental) conditions. As the rate constants of different individual processes may display very different sensitivity to experimental conditions, it is obvious that the conditions of the present experiment (the non-polar solvent, the narrow range of the temperatures, the low concentrations of acceptor) were the main factor which allowed (or forced) this electron-donor-acceptor system to display kinetics with the reversed meaning of decay parameters.

Finally, we should mention that NP-TRCNE system is capable to form ground-state-stable CT complexes (and the same is true for TRCNE systems with two other electron donors, i.e p-xylene and hexamethylbenzen). Apparently, the formation of CT complexes of NP-TRCNE does not interfere, under the conditions of the present experiment, with the kinetics of photoinduced reaction of exciplex formation, but this may drastically change with the change of solvent, quencher concentration, and at higher temperatures. It is also signalled by the observations of excitation spectra of $p$-xylene-TRCNE exciplex fluorescence (cf. discussion in Sec. 4.1) that the possibility of the existence of different orientational isomers of CT complex can be expected - a situation rather common for many EDA systems, in which both ways of creation of excited CT state, $\left(\mathrm{D}^{+} \mathrm{A}^{-}\right)^{*}$, are possible and observed in liquid solution (and/or in the gas phase). Their presence is usually of importance in the kinetics and dynamics of relaxation of excited Franck-Condon states of the system, different for different 
modes of excitations [19, 22, 36-39]. This aspect of the kinetics of EDA systems of TRCNE with aromatic hydrocarbon electron donors, which requires calculations of optimized structures for the ground- and excited-states orientational isomers, refinement of their electronic character and energetics, as well as the construction of relevant potential energy surfaces for the ground- and excited-states, is now under study.

\section{References}

[1] H. Leonhardt, A. Weller, Z. Phys. Chem. 29, 277 (1961).

[2] H. Leonhardt, A. Weller, Ber. Bunsenges. Phys. Chem. 67, 791 (1963).

[3] N. Knibbe, K. Röllig, F.P. Sächfer, A. Weller, J. Chem. Phys. 47, 1184 (1967).

[4] N. Mataga, T. Okada, N. Yamamoto, Chem. Phys. Lett. 1, 119 (1967).

[5] H. Beens, A. Weller, Acta Phys. Pol. A 94, 593 (1968).

[6] G. Jones II, in: Photoinduced Electron Transfer, Eds. M.A. Fox, M. Chanon, Part A, Elsevier, Amsterdam 1992, p. 245 (and references cited therein).

[7] Y. Hass, O. Anner, ibid. p. 305.

[8] M. Itoh, O. Kajimoto, in: Dynamics of Excited Molecules, Ed. K. Kuchitsu, Elsevier, Amsterdam 1994, p. 333.

[9] K. Wynne, G. Calli, R.M. Hochstrasser, J. Chem. Phys. 100, 4797 (1994).

[10] K. Wynne, G.D. Reid, R.M. Hochstrasser, J. Chem. Phys. 105, 2287 (1996).

[11] M. Hayashi, T.-S. Yang, J. Yu, A. Mebel, S.H. Lin, J. Phys. Chem. A 101, 4156 (1997).

[12] I.V. Rubtsov, K. Yosihara, J. Phys. Chem. A 101, 6138 (1997).

[13] M. Hayashi, T.-S. Yang, J. Yu, A. Mebel, R. Chang, S.H. Lin, I.V. Rubtsov, K. Yoshihara, J. Phys. Chem. A 102, 4256 (1998).

[14] R.E. Merrifield, W.D. Phillips, J. Am. Chem. Soc. 80, 2778 (1957).

[15] R.S. Mulliken, J. Am. Chem. Soc. 72, 600 (1950); 74, 811 (1952).

[16] R. Foster, Organic Charge-Transfer Complexes, Academic Press, London 1969.

[17] K. Kulinowski, I.R. Gould, N.S. Ferris, A.B. Myers, J. Phys. Chem. 99, 17715 (1995).

[18] J. Prochorow, Bull. Acad. Pol. Sci., Ser. Sci. Math. Astr. Phys. XV, 37 (1967).

[19] J. Prochorow, A. Tramer, J. Chem. Phys. 47, 775 (1967).

[20] J. Prochorow, J. Luminesc. 9, 131 (1974).

[21] T.D. Russell, D.H. Levy, J. Phys. Chem. 86, 2718 (1982).

[22] J. Prochorow, I. Deperasińska, J. Mol. Struct. 450, 47 (1998).

[23] N. Mataga, Y. Kanda, T. Asahi, H. Miyasaka, T. Okada, Chem. Phys. 127, 249 (1988).

[24] P. Bruni, G. Tosi, Gazetta Chim. Ital. 127, 435 (1997).

[25] Y. Shirota, I. Tsushi, H. Mikawa, Bull. Chem. Soc. Jpn. 47, 991 (1974).

[26] C.L. Dickinson, D.W. Wiley, B.C. McKusick, J. Am. Chem. Soc. 82, 6132 (1960). 
[27] P. Bruni, G. Tosi, unpublished results.

[28] J.B. Birks, Photophysics of A romatic Molecules, Wiley Interscience, London 1970. chaps. 4, 7, 9 .

[29] E. Gawęda, J. Prochorow, Chem. Phys. Lett. 30, 155 (1975).

[30] B. Stevens, M.I. Ban, Trans. Faraday Soc. 60, 1515 (1964).

[31] J. Dresner, J. Prochorow, W. Ode, J. Phys. Chem. 93, 671 (1989).

[32] J. Dresner, R. Kojer, J. Prochorow, I. Deperasińska, J. Luminesc. 71, 157 (1997).

[33] K.A. Zachariasse, in: Photochemistry on Solid Surfaces, in series Studies in Surface Science and Catalysis, Eds. M. Anpo, T. Matsuura, Vol. 47, Elsevier, Amsterdam 1989 , p. 48.

[34] N. Mataga, A. Ezumi, K. Takahashi, Z. Phys. Chem. N.F. 44, 250 (1965).

[35] B. Berlman, Handbook of Fluorescence Spectra of Aromatic Molecules, Academic Press, New York 1965, p.

[36] I. Deperasińska, J. Prochorow, J. Mol. Struct. 436, 585 (1997).

[37] J. Prochorow, J. Mol. Struct. 404, 199 (1997).

[38] I. Deperasińska, J. Prochorow, J. Dresner, J. Luminesc. 79, 65 (1998).

[39] I. Deperasińska, A. Sobieraj, Acta Phys. Pol. A 95, 309 (1999). 